\title{
Rapid Reconstruction of Tree Skeleton Based on Voxel Space
}

\author{
Gang Zhao, Yintao Shi, Maomei Wang and Yi Xu \\ JiangsuProvincialHydraulic Research Institute, Nanjing, China, 210017
}

\begin{abstract}
Tree skeleton had great significance in constructing the three-dimensional model of the botanical tree and investigating the forestry. Based on the point clouds of the botanical tree collected via the terrestrial $3 D$ laser scanner, we proposed a method of rapidly constructing the tree skeleton with the help of the voxel space conversion. We firstly constructed a specific voxel space according to the collected point clouds, and thenanalyzedthe connectivity components of each layer in voxel space and calculated the skeleton nodes contained in every voxel layer.At last, we constructed the whole skeleton of the botanical tree by means of single-source shortest path algorithm. Experiments show that the method proposed in this paper can be effectively.
\end{abstract}

Keywords-voxel space; skeleton; nodes; digraph

\section{INTRODUCTION}

Skeleton referred to original shapes connectivity and topology consistent light curve as an ideal expression of an object.It was first proposed by Blum, then known as skeleton as medial axis, later known as the axis (symmetric axis).Treeskeletons played an important role in the study of trees. It was not only the foundation of the tree three-dimensional reconstruction, but had an important meaning in the study of computer graphics, forestry and other fields.Tree skeletons continuously expanded the range of applications, but since limited by the highly irregular geometric shape and complex and unordered topological structure, the method of constructing accurate and efficient tree skeletons still had many issues to be further discussed.In this paper, we set the tree point cloud scanned by terrestrial 3D laser scanner as the source data, and proposed a new method of reconstructing tree skeleton based on voxel space.

\section{Method of Constructing Tree SKeleton BASED on VOXEL SPACE}

The proposedmethod of constructing tree skeletons based on voxel space implemented optimization and skeletonization primarily based on voxel space that was transformed from scanning Cartesian coordinate.First of all, based on the acquired point clouds, we builta voxel space, and then extracted the nodes of tree skeletons using digital image processing techniques.Finally,we used single-source shortest path algorithm to build tree skeletons.

\section{A. Constructing Voxel Space}

Voxel was the abbreviation of volume pixeland the smallest unit of digital information in three dimensional spaces, which itself did not contain location information in their coordinates, but rather from their position relative to other voxels to calculate location that they constituted a single volume image position in the data structure.A voxel space $\boldsymbol{T}$ consisted of a serial of voxel as a basic unit, and could be divided into three dimensions of Plane, Column andLine.The voxel coordinates could be represented as $t_{i} \in\left\{\left(l_{i}, c_{i}, p_{i}\right) \mid i=1, \cdots, N\right\}$, among whichl,c and prespectively represented the voxel location of Plane, Column and Line in voxel space shown in Figure I .The amounts of plane, columns and lines in a voxel space depended on the resolution selected by the space division as well as the maximum and minimum values of $\mathrm{x}, \mathrm{y}, \mathrm{z}$ coordinates in the point cloud data.

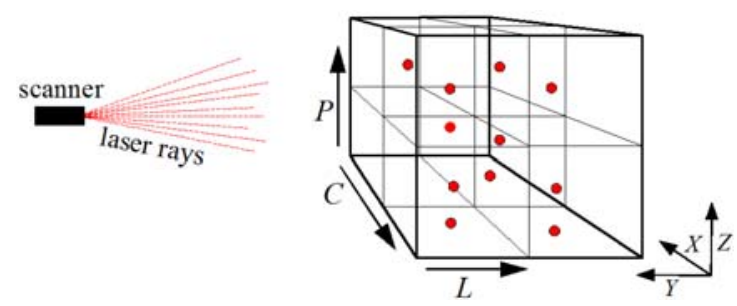

FIGURE I. CONSTRUCTED VOXEL SPACE

Based on the point cloud data obtained by terrestrial laser scanner, the constructing process of voxel space were as follows:

1) Calculate the boundary values

That boundary contained the entire point cloud data, and could be represented as $\left[x_{\min }, x_{\max }\right]$ 、 $\left[y_{\min }, y_{\max }\right]$ 、 $\left[z_{\min }, z_{\max }\right]$;

2) Select the appropriate resolution to divide the voxel space

The resolution solution was primarily based on a single voxel $\operatorname{size} v_{\text {size, }}$, and itsequation was as follows:

$$
\mathrm{v}_{\text {size }}=\sqrt[3]{\frac{\alpha \mathrm{k}}{\mathrm{n}} \Pi\left(\mathrm{f}_{\max }-\mathrm{f}_{\min }\right)}(\mathrm{f}=\mathrm{x}, \mathrm{y}, \mathrm{z})
$$

In Eq.(1), the number $\boldsymbol{n}$ was the number of the points contained in the voxel space, $\boldsymbol{\alpha}$ was a scalar adjusting the side length of a single voxel, $\boldsymbol{k}$ was the number of the closest points. When the edge length of the voxel unit was determined, the resolutions in the orientation of $\boldsymbol{L}, \boldsymbol{C}$ and $\boldsymbol{P}$ were as follows:

$$
\left\{\begin{array}{l}
\mathrm{l}=\left\lceil\left(\mathrm{x}_{\max }-\mathrm{x}_{\text {min }}\right) / \mathrm{v}_{\text {size }}\right\rceil \\
\mathrm{c}=\left\lceil\left(\mathrm{y}_{\max }-\mathrm{y}_{\min }\right) / \mathrm{v}_{\text {size }}\right\rceil \\
\mathrm{p}=\left\lceil\left(\mathrm{z}_{\max }-\mathrm{z}_{\min }\right) / \mathrm{v}_{\text {size }}\right\rceil
\end{array}\right.
$$


In Eq. (2), the operator $[\cdot$ |represented rounding upward.

\section{3) Partitionthe point clouds}

We partitioned the point clouds into the corresponding voxel coordinates in the voxel space, as shown in Figure I .

After the process described above, the point cloud data in Cartesian coordinates $(\boldsymbol{x}, \boldsymbol{y}, \boldsymbol{z})$ would be transformed into the voxel space $(\boldsymbol{l}, \boldsymbol{c}, \boldsymbol{p})$, as shown in Figure I. In the voxel space, the assigned value of each voxel was the number of the points in the space $(\boldsymbol{x}, \boldsymbol{y}, \boldsymbol{z})$.As popular known, the information that the point clouds described was the surface of the tree, and after space conversion, only the voxels intersected with the surface of the tree had non-zero value.

\section{B. Extracting Skeleton Nodes}

In order to extract the skeleton nodes of the tree, it was necessary converting the constructed voxel space to the binary voxel space which assumed that the voxel unit containing the point cloud data had a value of 1 , others had a value of 0 . On this basis, starting from the voxel layer of the tree root, extracted the skeleton nodes contained in each layer by layer from bottom to top.

Any layer of the constructed voxel space based on point clouds was projected onto a 2D planar space along the direction perpendicular to the ground, and one empty cell of one voxel width was added around the projection image, thus the extraction process of the skeleton nodes contained in this layer described as follows.

1) Define adjacency relations of 4-adjacent and 8-adjacency:

The former was defined as adjacency relations of current pixel in four directions of East (right), South (below), West (left) and North (upper), and the latter was in eight directions of East, South, West, North, Northeast, Southeast, Southwest and Northwestshown in Figure II.

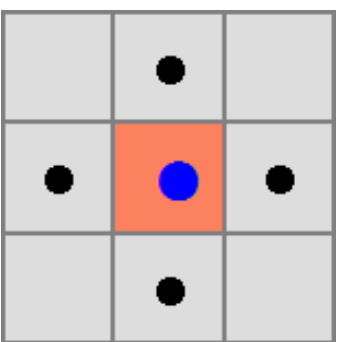

(A) 4-ADJACENT

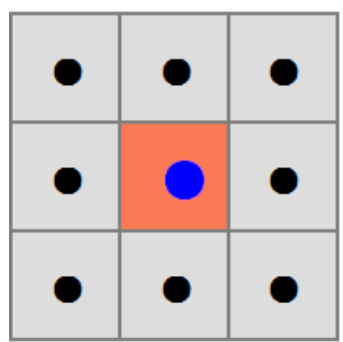

(B) 8-ADJACENT
FIGURE II. DEFINED ADJACENCY

2) Classify and mark.

Each layer of the voxel space represented the section information of the tree in different heights, and the voxels containing point clouds were represented by 1 , and other empty cells were represented by 0 . Different connected sets containing value 1 represented the different plane split of the tree branches, and in order to extract the skeleton nodes of each layer, it was necessary to separate the different branches.Firstly, we inverted the projection imagethat meant to convert all units containing value 1 to value -1 , and those containing value 0 remained unchanged. Then, based on line-rank scanning, we made the classification mark of the connected components in the projection image. That is to say, only labeled the units whose value were -1 , and ignored those valueswere 0.Label regular was judged by the neighborhood relation described as follows.First of all, we obtained the adjacent unit sets $\boldsymbol{P}$ closing to the current unit with the help of the neighborhood relation predefined above.Then, one by one searched each unit in the set $\boldsymbol{P}$ to judge if the number of labeled units was zero or not. If zero, the unit was assigned $\boldsymbol{L a b e l}=\boldsymbol{L a b e l}+\mathbf{1}$, otherwise it was labeled minimum label $\min (\boldsymbol{P})$ existing in the set $\boldsymbol{P}$.

\section{3) Merger}

After classification and label, the different connected components had been roughly split.But due to the search order, the units belonging to the same connected sets may be labeled as different symbols, so it required further merger. In the order from row to column, the analyses of every single cell in images were implemented one by one,and the label of the current unit was set as its smallest label in the 8-neighborhood sets. As shown in Figure III, (a) was the preliminary state of the projection image, (b) was the invention of the above image and (c) was the state after classification and merger.

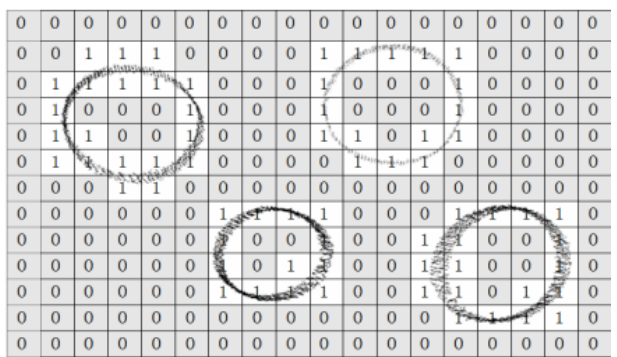

(a)

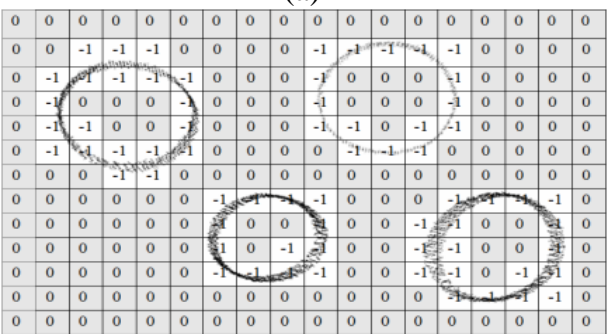

(b)

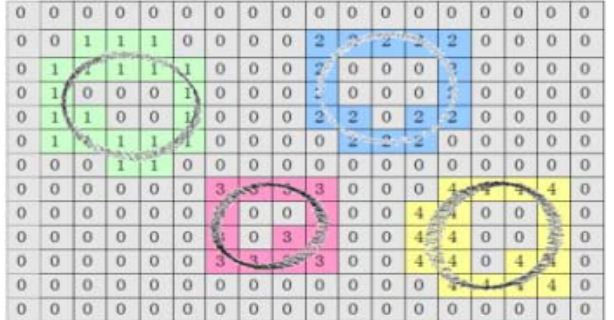

(c)

FIGURE III. LABEL CONNECTED COMPONENT

\section{4) Solve skeleton nodes}

According to the connected component analysis of projecting the voxel units onto the $2 \mathrm{D}$ planar image, the voxel units composing each branch section could be determined, and so were the connection relationship between those 
voxels.Presuming the point clouds that a connected component sets contained were $S=\left\{\left(x_{i}, y_{i}, z_{i}\right) \mid i=1,2, \cdots, n\right\}$,the Eq. (3) could be used to solve the skeleton node of certain branches cross-section.

$$
\left(\begin{array}{lll}
\bar{x} & \bar{y} & \bar{y}
\end{array}\right)^{T}=\left(\begin{array}{lll}
\frac{\sum_{i=1}^{\mathrm{n}} \mathrm{x}_{\mathrm{i}}}{\mathrm{n}} & \frac{\sum_{\mathrm{i}=1}^{\mathrm{n}} \mathrm{y}_{\mathrm{i}}}{\mathrm{n}} & \frac{\sum_{\mathrm{i}=1}^{\mathrm{n}} \mathrm{z}_{\mathrm{i}}}{\mathrm{n}}
\end{array}\right)^{\mathrm{T}}
$$

\section{Skeleton Construction}

After extracting the skeleton nodes of each layer, the connection relation between nodes were uncertain, particularly in the branches bifurcation. So it was crucial how to effectively identify the connections between nodes for correctly constructing tree skeletons. In this paper, one kind of single-source shortest path algorithm was applied to solve this problem that was based on the tree structure in Graph Theory. Further, firstly built a graph using the extracted nodes, and then rebuilt the whole skeleton in a weighted directed graph.

First of all, we selected all the extracted skeleton nodes as the vertex of the graph, and established a vertex collection of this graph $\boldsymbol{V}=\{1,2, \cdots, n\}$. Then, starting from the bottom of the voxel space, we connected each vertex in graph $\boldsymbol{V}$ with its adjacent vertex belonging to a certain range in upward direction, and simultaneouslyassigned each edge a right which was equivalent to the length of that edge. Thus far, a weighted directed graph $\boldsymbol{G}=(\boldsymbol{V}, \boldsymbol{E})$ could be established, among which $\boldsymbol{E}$ was the edge sets consisting of all vertexes in the set $\boldsymbol{V}$. Thereafter, the vertices collection $\boldsymbol{V}$ in the graph $\boldsymbol{G}$ was divided into two groups, one of which was a collection of vertices that had been solved the shortest path and the rest belonged to another group.Finally, we selected the root nodes of the tree as a source point, and determined the shortest path of the graph via a loop traversal, and thus the connection relation among the skeleton nodes could be determined. As shown in Figure IV,(a) was the collection of the scattered points, (b) was the constructed graph, (c) was the result of pathway search and (d) was the constructed skeleton.

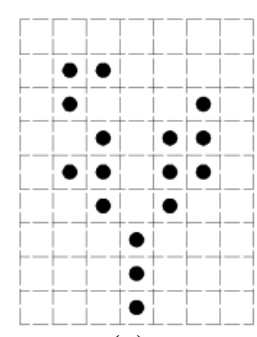

(a)

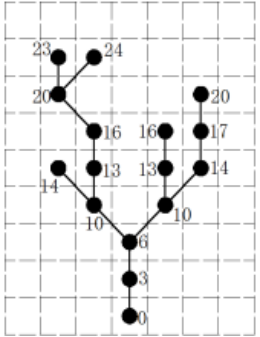

(c)

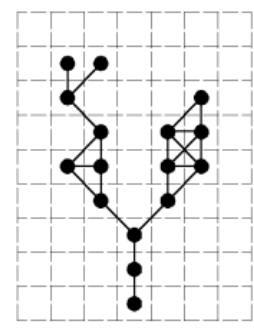

(b)

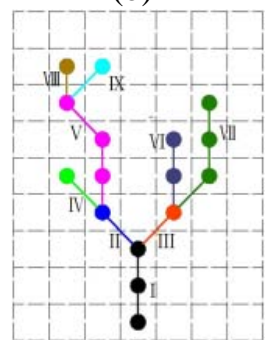

(d)
FIGURE IV. SKELETON CONSTRUCTION

\section{EXPERIMENTAL ANALYSIS}

In order to analyze the validity and reliability of the skeleton extraction method proposed in this paper, we had implemented a serial of experiment. Firstly, we obtained the point clouds of a tree by means of terrestrial 3D laser scanner, and then manually removed the point clouds of the leaves and retained those of the branches. Subsequently, we constructed the voxel space of the point clouds, and based on that, extracted the collection of the skeleton nodes.Finally, we reconstructed the whole skeleton of the tree via a kind of single source shortest path algorithm.Processing flow was shown in Figure $\mathrm{V}$, among which (a) was the realistic appearance of the tree, (b) was the point clouds of branches except for the leaves, (c) is the collection of the extracted skeleton nodes, and (d) is the whole reconstructed skeleton. The experiment testified that this proposed method was effective and feasible, and it could efficiently extract the skeleton from the point clouds.

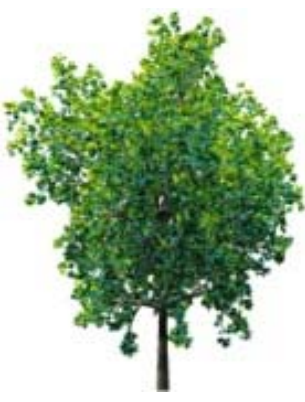

(a)

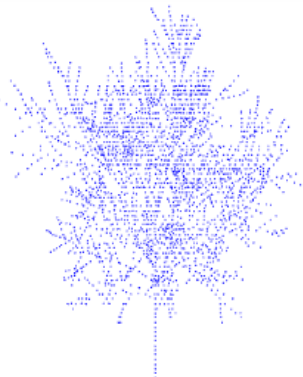

(c)

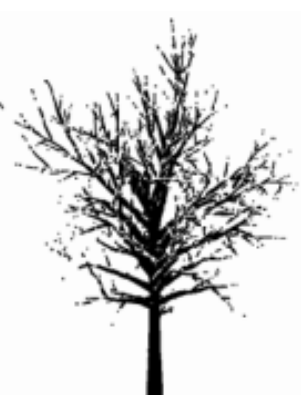

(b)

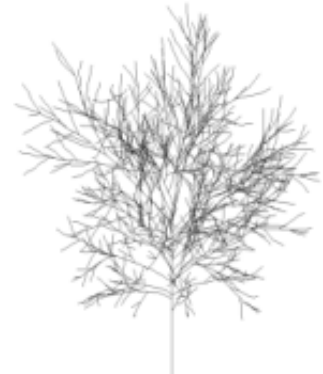

(d)
FIGURE V. EXPERIMENT

\section{CONCLUSION}

Three dimensional models of the realistic trees had been more and more popularly utilized in landscape design, environmental monitoring, Agriculture \& Forestry etc., and the skeletons of trees was the key to accurately construct 3D models of trees. The method proposed in this paper could not only solve the problem of tree skeleton extraction, but also had some reference for other skeleton extraction of complex and irregular objects.

\section{ACKNOWLEDGMENT}

This paper was funded by the research projects (JSCHKY201511) Jiangsu Provincial Bureau of Surveying, Mapping and Geoiformation afforded.

\section{REFERENCES}

[1] Runions A,Lane B,Prusinkiewicz P. Modeling trees with a space 
colonization algorithm[C]// Proceedings of Eurographics Workshop on Natural Phenomena2007. Pennsylvania: Penn State University Press, pp.63-70, 2007.

[2] Côté J F,Widlowski J L,Fournier R. A.,Verstraete M M. The structural and radiative consistency of three-dimensional tree reconstructions from terrestrial lidar[J]. Remote Sensing of Environment, Vol.113,A5, pp.1067-1081,2009.

[3] Tagliasacchi A,Zhang H. Curve skeleton extraction from incomplete point cloud[J]. ACM Trans. on Graphics, Vol.28, A3, pp.1-9, 2009.

[4] Li G,Liu L G,Zheng H.L,Mitra N J. Analysis, Reconstruction and Manipulation using Arterial Snakes[J]. ACM Transactions on Graphics, Vol.29, A6, pp.152-164, 2010.

[5] Cornea N D,Silver D,Min P. Curve-skeleton properties, applications, and algorithms[J]. IEEE Transactions on Visualization and Computer Graphics, Vol.13, A3, pp.530-548, 2007.

[6] Cheng Z L,Zhang X P,Chen B Q. Simple reconstruction of tree branches from a single range image[J]. Computer Science Technology, Vol.22,A6, pp.846-858, 2007. 